\title{
HLA-DR regulates macrophage phenotypic transformation and affects malignant behavior in esophageal squamous cell carcinoma
}

Jiang Fen Li

Shihezi University School of Medicine

Yu Fang Xie

Shihezi University School of Medicine

Wei Hua Liang

Shihezi University School of Medicine

Hai Jun Zhang

Shihezi University School of Medicine

\section{Xue Li Wang}

Shihezi University School of Medicine

Ji Hong Liu

Shihezi University School of Medicine

Kai Liu

Shihezi University School of Medicine

Xian Li Jiang

Shihezi University School of Medicine

Chen Hao Jiang

Shihezi University School of Medicine

Xin Yuan

Shihezi University School of Medicine

An Zhi Zhang

Shihezi University School of Medicine

Li Juan Pang

Shihezi University School of Medicine

Xiao Bin Cui

Shihezi University School of Medicine

Lan Yang

Shihezi University School of Medicine

Chun Xia Liu

Shihezi University School of Medicine

Wen Yi Gu

University of Queensland 


\section{Feng Li}

Capital Medical University

Jian Ming Hu ( $\square$ jianming_120@126.com )

Shihezi University School of Medicine

\section{Research}

Keywords: esophageal squamous cell carcinoma, tumor microenvironment, tumor-associated macrophages, phenotypic transformation, HLA-DR

Posted Date: August 3rd, 2020

DOl: https://doi.org/10.21203/rs.3.rs-49282/v1

License: @ (i) This work is licensed under a Creative Commons Attribution 4.0 International License. Read Full License 


\section{Abstract}

Background Tumor-associated macrophages (TAMs) are an important immune cell component of the tumor microenvironment. This study aimed to explore the molecular mechanism of TAMs phenotype transformation and the role in the development of esophageal squamous cell carcinoma (ESCC).

Methods Co-culture conditions were employed to determine the phenotypic effects of TAMs on ESCC cell biological behavior. Tumor metastasis related molecules VEGF-C and MMP-9 produced by TAMs was evaluated by qRT-PCR and western blot. Expression of HLA-DR was knocked down in TAMs in vitro to determine the effects on macrophage polarization and the biological behavior of ESCC. We determined whether co-injection with M2 TAMs and macrophages depletion affected tumor growth in vivo tumor challenge model. Associations between HLA-DR, TAM density, and clinical outcomes were evaluated in patients with ESCC.

Results TAMs in ESCC samples were found to closely reflect the M2 phenotype of TAMs, and exhibited low expression of HLA-DR. Which was involved in ESCC tumor invasion and metastasis. Low expression of HLA-DR positively correlated with high-density of M2 TAMs, indicating high invasiveness and poor prognosis in patients with ESCC. Downregulation of HLA-DR in TAMs led to additional M2-type TAM polarization and more VEGF-C and MMP-9 secretion, promoted the malignant transformation of ESCC.

Conclusions These results demonstrate that downregulation of HLA-DR promote the transformation of M2 TAMs, and participate in the invasion and metastasis of ESCC.

\section{Background}

The aggressive invasion and high metastatic capacity of esophageal squamous cell carcinoma (ESCC) are important factors contributing to the poor prognosis of this disease [1]. The occurrence and development of tumor from the participation of a series of complex factors, and the role of the surrounding tumor microenvironment is crucial [2].The tumor microenvironment of ESCC contains various inflammatory and immune cells, and tumor associated macrophages (TAMs) comprise the main component of the immune cell compartment [3]. TAMs can induce epithelial-mesenchymal transition (EMT) in ESCC cells to promote tumor invasion and metastasis [4-6]. Increased TAMs density in tumor tissue is a poor prognostic factor in patients with ESCC [7]. Macrophages can differentiate into two polarized states according to their function and phenotype: tumor-supportive (M2 type) macrophages and tumor suppressive (M1 type) macrophages [8]. Macrophages have functional plasticity and can alter their functional profiles in response to environmental stimuli. For example, when macrophages are exposed to lipopolysaccharide (LPS) or gamma interferon (IFN- $\gamma$ ), they are polarized to the M1 phenotype and exhibit antitumor activities. However, exposure of macrophages to Th2 cytokines, such as interleukin-4 (IL-4) and IL-13, results in polarization to an M2 phenotype [9]. Anti-CD47 treatment alone can shift the phenotype of macrophages toward an M1 subtype in vivo[10]. The regulation of macrophage phenotypes is the key to the role of macrophages in promoting or suppressing tumor progression. However, the molecular signals that regulate these phenotypes and the corresponding regulatory mechanisms remain to be elucidated. 
The expression of MHC class II proteins (human leukocyte antigen class II, HLA-II) on antigen-presenting cells (APCs) promotes CD4 + T-helper-mediated activation of B cells and cytotoxic CD8 + T cells [11]. Macrophages are a classic type of APC cells. Studies have shown that there is a significant negative correlation between the expression of HLA class II molecules (HLA-DR, DP, DQ) and CD68 + CD163 + TAMs, and low expression of HLA-II and high-density TAMs are associated with poor prognosis of cancer patients[12, 13]. HLA-DR as an important antigen of HLA-Il gene, is associated with the immune response to tumor cells, which can lead to poor outcome of tumor patients [14]. Suggested that HLA-DR is involved in the regulation of tumor progression, which may be mediated by TAMs, but the regulatory effect and mechanism have not been reported. In the present study, we evaluated the effects of HLA-DR in TAMs polarization and the impact of it on malignant behavior of ESCC.

\section{Results}

\section{TAMs promoted migration, and invasion of ESCC}

To understand the role of TAMs in the progression of ESCC, we co-cultured macrophages (PMA-treated THP-1) with ESCC cells (EC109 and EC9706). Compared with ESCC cells cultured alone, co-culture of ESCC with macrophages for $36 \mathrm{~h}$ altered cell morphology from the original round, oval-shaped morphology with epithelial characteristics to an elongated circle-shaped or spindle-shaped mesenchymal morphology, with enlarged gaps between cells, diffuse growth, and decreased adhesion (Fig. 1a). CCK8 and plate cloning experiments showed that TAMs did not significantly increase the proliferative capacity of ESCC cells (Fig. 1b-c). However, transwell assay with migration and invasion experiments demonstrated that TAMs significantly promote the migration and invasion of ESCC cells (Fig. 1d-e).

To interrogate the role of TAMs infiltration in ESCCs in vivo, we applied nude mice tumor formation experiment and human tissue for analysis. Tumor formation experiments in nude mice showed that the volume and weight of tumors from ESCC cells with chlorophosphate-depleted macrophages were significantly smaller than untreated group (Fig. 1f-h). We used human ESCC tissues for further verification, and found that increased number of tumor-infiltrating CD68 + TAMs was closely related to lymphatic invasion, lymph node metastasis, and clinical progression of ESCC $(P<0.05$, Fig. 1i). It suggested that there is a significant correlation between TAMs and tumor invasion and metastasis. In our previous research, we found that the VEGF-C in the VEGF family and MMP-9 in the tumor microenvironment have been proved possess a certain effect on ESCC metastasis[15].To further explore macrophage specific regulatory role on ESCC invasion, we evaluated expression of VEGF-C and MMP9 (considered to be key genes that promote tumor invasion and metastasis) in tumor tissue. We found that the expression of VEGF-C and MMP9 in ESCCs was significantly higher than that in normal tissues (Fig. 2a--b), and there was a significant higher expression of VEGF-C and MMP-9 in TAMs compare with no-co-culture group (Fig. 2c). Consistently, it was confirmed by qRT-PCR (Fig. 2d), suggesting that TAMs may promote the invasion and metastasis of ESCC, which is closely related to VEGF-C and MMP9 secreted by TAMs.

\section{M2 macrophages were the primary TAM phenotype that promoted malignant behavior of ESCC cells}


Macrophages are generally classified as M1 or M2 macrophages [16, 17]. Immunofluorescence (IF) imaging was used to evaluate changes in macrophage phenotypes in a co-culture system, and found that the number of CD163-positive M2-type TAMs was significantly increased following co-culture with ESCC cells, while the number of M1-type macrophages (indicated by co-staining of HLA-DR and CD68) was significantly reduced (Fig. 3a-b). We further evaluated macrophage phenotypes in vitro by qRT-PCR, and found that macrophages co-cultured with ESCC cells exhibited molecular characteristics of M2 phenotype macrophages, including significantly increased IL-10 expression and decreased TNF- $a$ and HLA-DR expression (Fig. 3c). These data suggest that there is phenotypic transformation of macrophages in the ESCC microenvironment, and M2 TAMs may be the main component of macrophages in ESCC microenvironment.

Nude mice in Tumor formation experiments, we induced macrophages to an M2 phenotype in vitro, then coinjected these M2 macrophages along with ESCC cells as subcutaneous tumors. Found that tumors were larger and heavier in the M2 TAMs co-culture ESCC cell group compared with the ESCC cell-alone injection group (Fig. 3d-f). Further verification in human ESCC tissues, we found that the increase in the number of infiltrating CD163-positive M2 TAMs was closely related to lymphatic invasion, lymph node metastasis, and clinical progression in patients with ESCCs (Fig. $3 g$ ). It is suggests that M2 macrophages are the primary TAMs phenotype that promotes malignant behavior of ESCC cells

\section{Hla-dr Mediated Macrophage Phenotypic Transformation}

To screen for molecular mechanisms that may be related to the M2 polarization of macrophages, we analyzed microarray data (dataset GSE95405 from the GEO database) to compare gene expression between THP-1 cells that were induced into M1 macrophages by treatment with phorbol ester (PMA), lipopolysaccharide (LPS) and IFN- $y$, and THP-1 cells that were induced into M2 macrophages by treatment with IL-4 and IL-13 (Fig. 4a). Significant differences in the expression of multiple members of the HLA-DR family between M1 and M2 type macrophages were identified. Compared with M1-type macrophages, M2type macrophages expressed less HLA-DR (Fig. 4b). To address whether HLA-DR downregulation is a key molecular event involved in the transformation of macrophages into a cancer-promoting M2 phenotype, we transfected macrophages with HLA-DR lentiviral shRNA. HLA-DR expression in macrophages was significantly lower after shRNA transfection (Fig. 4c-e). Silencing HLA-DR significantly increased expression of the macrophage molecular marker IL-10, and significantly reduced expression of TNF-a (Fig. 4f). These data demonstrate that downregulation of HLA-DR promoting the macrophages polarization to M2 phenotype.

\section{Effects of HLA-DR knockdown on TAM-mediated malignant behavior of ESCC}

To investigate the effects of HLA-DR knockdown in macrophages on the biological behavior of ESCC tumor cells, we co-cultured HLA-DR knockdown macrophages and ESCC cells in vitro, and found that HLA-DR knockdown macrophages significantly increased the proliferation of ESCC cells compared with control macrophages $(P<0.05$, Fig. 5a). Transwell migration and invasion assays showed that HLA-DR knockdown macrophages significantly promoted the migration and invasion of ESCC cells $(P<0.05$, Fig. $5 b-c)$. In addition, we found that knockdown of HLA-DR significantly increased the expression of VEGF-C and MMP-9 
in TAMs $(P<0.05$, Fig. $5 \mathrm{~d}-\mathrm{e})$, suggesting that downregulation of HLA-DR in macrophages induces TAM M2 phenotypic transformation, promotes secretion of VEGF-C and MMP-9, and participates in the malignant transformation of ESCC.

\section{Low expression of HLA-DR positively correlated with high-density of M2 TAMs, indicating high invasiveness and poor prognosis in ESCC}

Our experiments in vitro demonstrated that downregulation of HLA-DR contributes to macrophage M2 polarization and promotes malignant behavior of ESCC cells. To further verify the pro-cancer effect of HLADR downregulation, the expression of HLA-DR in clinical ESCC samples was evaluated, and found that HLADR is mainly expressed in stromal cells in ESCC, and that macrophages constitute an important population of HLA-DR expressing cells (Fig. 6a). Moreover, we observed that some CD68-positive TAMs did not express HLA-DR, suggesting that some TAMs may have lost HLA-DR, and represent a group of M2 phenotype TAMs (Fig. 6a). Identification of M2 TAMs with CD163 staining revealed that ESCC areas with low levels of HLADR expression contained high densities of M2 TAMs. Furthermore, in areas with high expression of HLA-DR, there were fewer M2 TAMs (Fig. 6b). Comparing the distribution of M2 TAMs with clinicopathological information, we found that the lower expression of HLA-DR was positively correlated with an increase in the number of M2 TAMs, and positively correlated with invasion depth, lymph node metastasis, and clinical stage of ESCC (Table 1). Analysis of gene expression from the TCGA database and the prognostic data from patients in this study, revealed that lower expression of HLA-DR was closely related to poor prognosis (Fig. 6c-d). These data suggest that lower expression of HLA-DR may be related to macrophage transformation to an M2 phenotype and associated with increased invasiveness and poor prognosis of ESCC. 
Table 1

Correlation between HLA-DR expression and clinicopathological parameters in esophageal squamous cell carcinoma (ESCC) tissues.

\begin{tabular}{|lllll|}
\hline Variable & Cases $(\mathrm{N})$ & HLA-DR & HLA-DR & P-value \\
\cline { 3 - 5 } & & Negative $(0,1)$ & Positive $(2,3)$ &
\end{tabular}

Age (y)

\begin{tabular}{lllll}
$\mathbf{y}$ Median (58y) & 81 & $23(28.4 \%)$ & $58(71.6 \%)$ & 0.868 \\
\cline { 1 - 3 } Median & 59 & $16(27.1 \%)$ & $43(72.9 \%)$ &
\end{tabular}

Gender

$\begin{array}{|lllll|}\text { Male } & 96 & 30(31.3 \%) & 66(68.8 \%) & 0.226 \\ \text { Female } & 44 & 9(20.5 \%) & 35(79.5 \%) & \end{array}$

Tumor location

\begin{tabular}{|c|}
\hline Upper \\
\hline
\end{tabular}

$\begin{array}{llll}\text { Middle } & 109 & 35(32.1 \%) & 74(67.9 \%) \\ \text { Lower } & 96 & 4(13.8 \%) & 25(86.2 \%)\end{array}$

Histologic grade

\begin{tabular}{|c|c|c|c|}
\hline Well & 42 & $12(28.6 \%)$ & 30 (71.4\%) \\
\hline Moderate & 68 & 18 (26.5\%) & 50 (73.5\%) \\
\hline Poor & 30 & $9(30.0 \%)$ & $21(70.0 \%)$ \\
\hline
\end{tabular}

Depth of invasion

$\begin{array}{lllll}\text { T1-T2 } & 47 & 7(14.9 \%) & 40(85.1 \%) & 0.026 * \\ \text { T3-T4 } & 93 & 32(34.4 \%) & 61(65.6 \%) & \end{array}$

Venous invasion

$\begin{array}{lllll}\text { Negative } & 44 & 9(20.5 \%) & 35(79.5 \%) & 0.263 \\ \text { Positive } & 96 & 30(31.3 \%) & 66(68.8 \%) & \end{array}$

Nodal status

$* \mathrm{P}<0.05,{ }^{*} \mathrm{P}<0.01$, *** $\mathrm{P}<0.001$ 


\begin{tabular}{|c|c|c|c|c|}
\hline \multirow[t]{2}{*}{ Variable } & \multirow[t]{2}{*}{ Cases $(\mathrm{N})$} & HLA-DR & HLA-DR & \multirow[t]{2}{*}{$P$-value } \\
\hline & & Negative $(0,1)$ & Positive $(2,3)$ & \\
\hline $\mathrm{pN}-$ & 75 & $13(17.3 \%)$ & $62(82.7 \%)$ & $0.005^{\star \star *}$ \\
\hline $\mathrm{pN}+$ & 65 & $26(40.0 \%)$ & $39(60.8 \%)$ & \\
\hline \multicolumn{2}{|c|}{ TNM stage } & & & \\
\hline I-II & 91 & 15 (16.5\%) & 76 (83.5\%) & $P<0.001^{\star \star \star}$ \\
\hline III-IV & 49 & $24(49.0 \%)$ & 25 (51.0\%) & \\
\hline
\end{tabular}

\section{Discussion}

ESCC contains a large number of TAMs, which constitute an important immune cell population within the tumor microenvironment. TAMs play a key role in tumor progression by secreting a variety of cytokines, chemokines, and angiogenic factors [18]. TAMs have been reported to be related to the malignant behavior of tumors and to be important factors leading to poor prognosis in a variety of tumors, such as pancreatic ductal carcinoma and breast cancer [19-21]. It is crucial to further define the mechanisms of TAM phenotypic transformation and their cancer-promoting mechanisms.

In our study, co-culture of macrophages and tumor cells did not significantly change the proliferation of ESCC cells, but did significantly enhance the invasion and migration of ESCC cells. In vivo tumorigenesis experiments in nude mice further confirmed that depletion of TAMs by chlorophosphite treatment significantly inhibited tumor growth. These results are similar to a study by Fan et al, in which it was reported that TAMs can promotes tumorigenicity, tumor invasion and metastasis [22]. Our results further support the pro-tumor role for TAMs, as our analysis of human ESCC tissue specimens, revealed that the density of CD68 + TAMs in ESCC was positively related to vascular infiltration and lymph node metastasis. This phenomenon has also been reported in variety of other tumors[23]. But specific mechanisms by which TAMs promote the development of ESCC still need to be further explored. MMP-9 and VEGF have been investigated as important factors related to invasion and metastasis in tumors[24]. In this study, VEGF-C and MMP-9 were found to be highly expressed in ESCC, and TAM was an important source of them compared to normal macrophages. These indicated that TAM is an important factor in promoting the invasion and metastasis of ESCC, mechanistically due to its production of VEGF-C and MMP-9. It is similar to Li et al report that, the induction of VEGF and MMP9 expression by macrophages from the tumor microenvironment is also one of the important sources [25].

Previous studies have found that the TAMs in ESCCs are mainly M2 type. M2 TAMs are considered to have low antigen delivery capacity, which may facilitate immunosuppression and promote tumor vascular growth and lymph node metastasis [26]. In the present study, immunofluorescence and qRT-PCR were used to evaluate changes in TAM phenotypes in a co-culture system with ESCC cells. This study demonstrated 
that the number of CD163-positive M2-type TAMs was significantly increased following co-culture with ESCC cells, while the number of M1-type TAMs was significantly reduced, suggesting that M2 phenotype macrophages play an important role in ESCC. In order to more fully explore the effects of M2 TAM on ESCCs, we induced macrophages into M2 TAMs by co-culture with ESCC cells. We then injected the M2 macrophages subcutaneously in nude mice, either alone or co-innoculated with ESCC cells. In this coinnoculation experiment, we found M2 TAMs significantly promoted the growth of ESCC tumors. Moreover, our results further suggest that M2 TAMs have a significant correlation with tumor metastasis and clinical progress in human ESCCs.

The regulation of macrophage phenotypes is key to the role of macrophages in promoting or suppressing tumor progression. To screen for molecular mechanisms that may be related to the M2 polarization of macrophages, we analyzed microarray data (dataset GSE95405 from the GEO database), and found that there are significant differences in the expression of multiple members of the HLA-DR family between M1 and M2 macrophages. And HLA-DR as a key marker has been used to distinguish M1 macrophages from M2 macrophages [27, 28]. Suggest HLA-DR deletion may be a molecular mechanism that mediates M2 TAM-mediated immune escape, but a causal relationship between loss of HLA-DR on M2 TAMs and immune escape of ESCC remains to be shown. So next HLA-DR lentiviral shRNA was used to downregulate macrophage HLA-DR expression, the results demonstrate that Macrophage was co-cultured with EC109 and EC9706 and exhibited a mixed M1 / M2 phenotype, while down-regulation of HLA-DR can further polarize macrophages to the M2 phenotype. Recent reports also showed the role of HLA in regulating macrophage phenotype $[29,30]$. Suggesting the loss of macrophage HLA-DR mediates immune escape and is an upstream molecular event that leads to M2 macrophage polarization.

In the co-culture system, we found that HLA-DR knockdown macrophages significantly increased the proliferation, migration and invasion of ESCC cells. In addition, we found that knockdown of HLA-DR significantly increased the expression of VEGF-C and MMP-9 in TAMs, suggesting that downregulation of HLA-DR in macrophages induces TAM M2 phenotypic transformation, promotes secretion of VEGF-C and MMP-9, and participates in the malignant transformation of ESCC.

Human ESCC tissue samples showed that HLA-DR was negatively correlated with the number of M2 type TAMs. This supports the findings from our in vitro experiments. We also demonstrated that low expression of HLA-DR in ESCC tumor tissues was associated with low survival rates of ESCC patients. This is consistent with previous studies, which report that low HLA-DR expression is a risk factor for poor prognosis in various tumors [31,32]. Therefore, we believe that the low expression of HLA-DR has predictive value for the recurrence and metastasis of ESCC. The expression of HLA-DR could be used as an important biomarker for the recurrence and metastasis of ESCC.

\section{Conclusion}

In summary, TAMs is critical factor that influence ESCC tumor formation, and the migration and invasion of ESCC cells. M2 type TAMs are a critical tumor microenvironment component that promote the malignant growth of ESCC. Moreover, downregulation of HLA-DR induces a transformation of macrophages into M2- 
type TAMs, resulting in enhanced production of VEGF-C and MMP-9, which may represent an important molecular mechanism by which M2 TAMs promote ESCC invasion and metastasis.

\section{Materials And Methods}

\section{Patients and immunohistochemistry}

All participants were patients at the Xinjiang Yili Friendship Hospital, China. Prior to participating in this study, each participant provided written informed consent. The Institutional Ethics Committee of the Erie Friendship Hospital ratified these protocols in accordance with the Declaration of Helsinki. From 2008 to 2014, tissue samples were collected from 140 cases of Xinjiang Kazakh (Kazakh) ESCC from Xinjiang Yili Kazakh Autonomous Prefecture Friendship Hospital by surgical resection for formalin fixation and paraffin embedding. According to NCCN guidelines, pathologists determined the tumor stage. The case information is shown in Supplementary Table 1

The survival status of all participants was followed by telephone contact until December 2015. The median follow-up time for living patients was 30 months (range 1-84 months). Overall survival (OS) was defined as the interval between surgery and death, or between surgery and the last patient follow-up. Of the 140 patients recruited, $73(52.14 \%)$ remained alive and 57 (47.86\%) died during the follow-up interval.

Monocytes immobilized with formalin can be induced to differentiate into macrophages after $36 \mathrm{~h}$, and can be co-cultured with cancer cells after $36 \mathrm{~h}$. After $36 \mathrm{~h}$, cells were incubated with diluted primary antibody (see below) overnight at $4^{\circ} \mathrm{C}$. Cells were then incubated with secondary antibody (1:50 dilution) for $30 \mathrm{~min}$. Cell nuclei were counterstained with 4,6-diamino-2-phenylindole for $15 \mathrm{~min}$. Images were obtained using a Leica SP5 spectral confocal microscope or an AMG EVOS FL microscope. The primary antibodies used were: for IF single staining: anti HLA-DR (1:50), anti-cd163 (1:50), for IF double staining: anti CD68 (1:200) (Abcam, ab5076, 1:50). Staining was observed and images were recorded using a fluorescence microscope.

\section{Cell culture}

The human ESCC cell lines EC109 and EC9706 were purchased from the American Type Culture Collection (ATCC) and cultured in RPIM-1640 (Gibco) containing 10\% fetal bovine serum (FBS, Gibco). Human THP-1 cells were purchased from ATCC and cultured in RPMI-1640 medium containing 10\% FBS. THP-1 cells were polarized into macrophages using PMA treatment (Sigma, $5 \mathrm{nM}$ ). Briefly, THP-1 cells were inoculated into a 6-well plate Transwell chamber at $2 \times 10^{6} \mathrm{cells} / \mathrm{ml}$, and cultured with RPMI- 1640 medium ( $10 \%$ serum) containing $200 \mathrm{ng} / \mathrm{ml}$ PMA for $24 \mathrm{~h}$. The ESCC cell lines EC109 and EC9706 were inoculated into the upper chamber of a 6-well plate chamber at $4 \times 10^{5} \mathrm{cells} / \mathrm{ml}$, and $2 \mathrm{ml} /$ well of complete DMEM were added to the upper and lower chambers. The cells were allowed to adhere for $12 \mathrm{~h}$. Macrophages and ESCC cell lines cocultivation was established bythe non-contact co-culture transwell system (Corning, USA). Inserts containing THP-1 induced macrophages were transferred to 6-well plate seeded with ESCC cells $\left(1 \times 10^{5}\right.$ 
cells per well) in advance and co-cultured, and the medium was replaced with fresh RPMI 1640 complete medium. Cells were incubated in a $5 \% \mathrm{CO}_{2}$ cell incubator at $37^{\circ} \mathrm{C}$ for $36 \mathrm{~h}$, and then used for experiments.

\section{Conditioned medium}

THP-1 cells were transformed into macrophages by induction with RPMI- 1640 medium ( $10 \%$ serum) containing $200 \mathrm{ng} / \mathrm{ml} \mathrm{PMA}$ for $24 \mathrm{~h}$. ESCC cells were placed in a Transwell cell and cultured with the macrophages for $36 \mathrm{~h}$. The cell culture medium of the upper and lower chambers was drawn into centrifuge tubes, centrifuged at $800 \mathrm{rpm}$ for $5 \mathrm{~min}$, and the supernatant was collected as conditioned medium and preserved at $-20^{\circ} \mathrm{C}$.

\section{Morphological observation of ESCC cells}

Co-cultured and monocultured ESCC cells were observed under a microscope. Images were taken with an Olympus imaging system in a $100 \times$ and $200 \times$ field.

\section{Cell proliferation experiment}

Cell proliferation was evaluated using the CCK8 assay. Briefly, $10 \mu \mathrm{l} \mathrm{CCK8}$ working buffer were added to each well of a 96 well plate, and absorbance was measured at $450 \mathrm{~nm}$ using a TECAN infinite m200 Pro enzyme scale.

\section{RNA extraction and quantitative real-time PCR (qRT-PCR)}

Total RNA was isolated using Trizol reagent. Total RNA was reverse transcribed with a Takara PrimeScript ${ }^{\mathrm{TM}}$ RT-PCR kit and amplified using a SYBR Premier ex Taq ${ }^{\text {TM }}$ II Kit (Takara), according to the manufacturer's instructions. All primers used are listed in Table S1. PCR was carried out using the eco (Illumina) system with parameters: initiation at $95^{\circ} \mathrm{C}$ for $30 \mathrm{~s}$, followed by 40 thermal cycles at $95^{\circ} \mathrm{C}$ for $3 \mathrm{~s}, 55^{\circ} \mathrm{C}$ for $30 \mathrm{~s}$ and $72^{\circ} \mathrm{C}$ for $30 \mathrm{~s}$, then $95^{\circ} \mathrm{C}$ for $15 \mathrm{~s}, 65^{\circ} \mathrm{C}$ for $60 \mathrm{~s}$, and $95^{\circ} \mathrm{C}$ for $15 \mathrm{~s}$. All data were analyzed by CT value comparison.

\section{Animal models}

Six-week-old BALB/c nude mice were purchased from the Shanghai Experimental Animal Center of the Chinese Academy of Sciences, and were maintained in a pathogen free environment. Thirty mice were randomly divided into three groups. Mice received subcutaneous injection of cell suspension of $1 \times 10^{6}$ EC109 cells into the left armpit; one group was also injected with $1 \times 10^{6} \mathrm{M} 2$ TAM cells. When the diameter of the transplanted tumor reached $0.2-0.4 \mathrm{~cm}$, chlorophosphate liposomes and phosphate-buffered saline (PBS) control liposomes (Fu Sheng Biotechnology Co, Ltd, China) were injected into the nude mice in the corresponding groups every 4 days until the end of the experiment. The mice were euthanized by cervical dislocation 4 weeks after injection, and the tumors were isolated for analysis.

\section{Plate clone formation assay}


EC109 and EC9706 cells (about $1 \times 10^{3}$ cells) co-cultured with macrophages were inoculated into a six-well culture dish. After incubation at $37^{\circ} \mathrm{C}$ for 2 weeks, the cells were washed twice with PBS and stained with $0.1 \%$ crystal violet solution. The colonies containing $\sim 50$ cells were counted under a microscope.

\section{Cell migration and cell invasion assays}

About $5 \times 10^{4}$ EC109 and EC9706 cells from the different co-culture groups were resuspended in $200 \mu$ l serum-free medium and inoculated into the upper chamber of a Transwell invasion insert, and $500 \mu \mathrm{l}$ medium supplemented with 10\% FBS were added to the lower compartment. After $24 \mathrm{~h}$ of culture, the cells that had migrated to the lower surface of the membrane were fixed with $4 \%$ paraformaldehyde, stained with $0.1 \%$ crystal violet, and counted under a microscope. These experiments were performed three times.

\section{Immunofluorescence assay}

Cells grown in 6-well plates were fixed for $20 \mathrm{~min}$ in $4 \%(\mathrm{w} / \mathrm{v})$ paraformaldehyde at room temperature, washed 3 times with PBS, and treated with 0.5\% (v / v) Triton X-100 (Solarbio, T8200) for 20 min and then blocked for 30 min with 5\% ( $/$ / v) normal serum in PBS. After that, cells were induced with primary antibodies at $4{ }^{\circ} \mathrm{C}$ overnight and secondary antibodies(1:50) for $1 \mathrm{~h}$ at room temperature, followed by washing and staining with DAPI (2 $\mathrm{gg} / \mathrm{ml}$, Solarbio, c0065).

\section{Western blotting}

Western blotting was performed as previously described [33]. Protein was extracted from TAM cells with RIPA lysis buffer (Beyotime, Haimen, China) containing protease inhibitor PMSF (CW Biotechnology, Beijing, China). Primary antibodies to VEGF-C (1:1000; Santa Cruz), MMP-9 (1:500; Santa Cruz), and HLA-DR (1:2000; Santa Cruz) were used. These antibodies were detected using goat polyclonal anti rabbit IgG antibody (1:10000; Abcam). Protein bands were detected using ECL.

\section{Construction of the lentivirus shRNA vector}

Human HLA-DR specific shRNA carrier and non-targeting (shnt) vector were purchased from Jikai Company of China .

Oligonucleotide sequence information: 


\begin{tabular}{|c|c|c|c|c|c|}
\hline No. & $5^{\prime}$ & STEM & Loop & STEM & $3^{\prime}$ \\
\hline $\begin{array}{l}\text { HLA-DRA- } \\
\text { RNAi(55452- } \\
\text { 1)-a }\end{array}$ & Ccgg & CCATCTTCATCATCAAGGGAT & CTCGAG & ATCCCTTGATGATGAAGATGG & TTTTTg \\
\hline $\begin{array}{l}\text { HLA-DRA- } \\
\text { RNAi(55452- } \\
\text { 1)-b }\end{array}$ & aattcaaaaa & сCATCTTCATCATCAAGGGAT & CTCGAG & ATCCСTTGATGATGAAGATGG & \\
\hline $\begin{array}{l}\text { HLA-DRA- } \\
\text { RNAi(55453- } \\
\text { 1) -a }\end{array}$ & Ccgg & gcTTGAAGAATTTGGACGATT & CTCGAG & AATCGTCCAAATTCTTCAAGC & TTTTTg \\
\hline $\begin{array}{l}\text { HLA-DRA- } \\
\text { RNAi(55453- } \\
\text { 1)-b }\end{array}$ & aattcaaaaa & gcTTGAAGAATTTGGACGATT & CTCGAG & AATCGTCCAAATTCTTCAAGC & \\
\hline $\begin{array}{l}\text { HLA-DRA- } \\
\text { RNAi(55454- } \\
\text { 1) -a }\end{array}$ & Ccgg & ctGACCAATCAGGCGAGTTTA & CTCGAG & TAAACTCGCCTGATTGGTCAG & TTTTTg \\
\hline $\begin{array}{l}\text { HLA-DRA- } \\
\text { RNAi(55454- } \\
\text { 1)-b }\end{array}$ & aattcaaaaa & ctGACCAATCAGGCGAGTTTA & CTCGAG & TAAACTCGCCTGATTGGTCAG & \\
\hline
\end{tabular}

\section{Microarray analysis from bioinformatics database}

The Oncomine database (https://www.oncomine.org/resource/login.html) was used to analyze the expression of VEGF and MMP-9 in ESCC and normal tissues [34]. Microarray data were obtained from Gene Expression Omnibus (Geo, http://www.ncbi.nlm.nih.gov/geo) from the GSE95405 study. GSE95405 microarray data included gene expression data from M0 and M1 macrophages from six cases. The expression of HLA-DR-related molecules was analyzed from this dataset. Data Were $\log _{2}$ transformed and the resulting heatmap was constructed using hemi software [35].

To further explore the expression and prognostic value of genes of interest in ESCC, we used a newly developed interactive website, ualcan, (http://ualcan.path.uab.edu/analysis. HTML) to analyze ESCC gene expression data from the TCGA database [36].

\section{Statistical analysis}

Statistical analysis was performed using SPSS 20.0 software (Chicago, IL, USA).. Measurement data between two groups were compared using paired tests and/or independent sample t-tests. Measurement data between three groups were compared using single factor analysis of variance. A difference was considered to be statistically significant at $P<0.05$.

\section{Abbreviations}

ESCC: Esophageal squamous cell carcinoma; TAMs: Tumor-associated macrophages; HLA-Il : Human leukocyte antigen class II; APCs: Antigen-presenting cells; qRT-PCR: Quantitative real-time PCR; IF: Immunofluorescence; PMA: Phorbol ester; LPS: Lipopolysaccharide; IL-4: Interleukin-4; IL-13: Interleukin-13; IFN- $y$ : interferon; FBS: Fetal bovine serum; PBS: Phosphate-buffered saline; GEO: Gene expression omnibus; OS :Overall survival 


\section{Declarations}

\section{Ethics approval and consent to participate}

All participants were patients at the Xinjiang Yili Friendship Hospital, China. Prior to participating in this study, each participant provided written informed consent. The Institutional Ethics Committee of the Erie Friendship Hospital ratified these protocols in accordance with the Declaration of Helsinki.All animal experiments were conducted according to protocols approved.

\section{Consent for publication}

Not applicable.

\section{Availability of data and materials}

All data generated or analyzed during this study are included in this published article .

\section{Competing interests}

The authors declare that they have no competing interests.

\section{Funding}

This work was supported by grants from the National Natural Science Foundation of China (No. 81460363, No. 81760428 and No. 81960435), the Start-up Project of High-level Talents Scientific Research in Shihezi University (RCZK2018C19), the Science and Technology Development Project of Xinjiang Production and Construction Corps (No. 2018AB033), the National Early Detection and Treatment Project for Upper Digestive Tract in Rural Area in China (No. 2018), and The Youth Science and Technology Innovation Leading Talents Project of Corps (No. 2017CB004).

\section{Authors' contributions}

JF,YF performed the experiments, analyzed the data and wrote the manuscript.WH, $H J, X L, J H, L K, X L$ did the experiments and analyzed the data. $\mathrm{CH}, \mathrm{XY}, \mathrm{AZ}$ did some experiments and provided useful helps.

$L J, X B, L Y, C X, W Y$ gave valuable suggestions. FL,JM designed and supervised the study, explained the data and wrote the manuscript. All authors read and approved the final manuscript.

\section{Acknowledgements}

Not applicable.

\section{References}

[1] E. Simon, D. Martin, L. Johan, W. Bengt, B.M. Daniel, S. Magnus, B. Michael. Esophageal cancer: current and emerging therapy modalities. Expert Rev Anticanc. 2008; 8: 1433-1448. 
[2] B. Ruffell, N.I. Affara, L.M. Coussens. Differential macrophage programming in the tumor microenvironment. Trends Immuno. 2012;33: 119-126.

[3] J.W. Pollard. Tumour-educated macrophages promote tumour progression and metastasis. Nat Rev Cancer. 2004;4:71-78.

[4] A.K. Bonde, V. Tischler, S. Kumar, A. Soltermann, R.A. Schwendener. Intratumoral macrophages contribute to epithelial-mesenchymal transition in solid tumors. BMC Cancer.2012;12:35.

[5] C.Y. Liu, J.Y. Xu, X.Y. Shi, W. Huang, J.L. Ding. M2-polarized tumor-associated macrophages promoted epithelial-mesenchymal transition in pancreatic cancer cells, partially through TLR4/IL-10 signaling pathway. Lab Invest. 2013;93:844-854.

[6] W.-j. Zhang, X.-h. Wang, S.-t. Gao, C. Chen, X.-y. Xu, Q. sun, Z.-h. Zhou, G.-z. Wu, Q. Yu, G. Xu. Tumorassociated macrophages correlate with phenomenon of epithelial-mesenchymal transition and contribute to poor prognosis in triple-negative breast cancer patients. J Surg Res. 2018;222:93-101.

[7] S. Keijiro, M. Hiroshi, T. Koji, T. Tsuyoshi, K. Yukinori, Y. Makoto, N. Kiyokazu, T. Shuji, M. Masaki, D. Yuichiro. High infiltration of tumor-associated macrophages is associated with a poor response to chemotherapy and poor prognosis of patients undergoing neoadjuvant chemotherapy for esophageal cancer. J Surg Oncol. 2015;111:752-759.

[8] R. Ostuni, F. Kratochvill, P.J. Murray, G. Natoli. Macrophages and cancer: from mechanisms to therapeutic implications. Trends Immunolo.2105; 36: 229-239.

[9] M.R. Galdiero, E. Bonavita, I. Barajon, C. Garlanda, S. Jaillon. Tumor associated macrophages and neutrophils in cancer, J Cell Physiol. 2013;218:1402-1410.

[10] M. Zhang, H. Gregor, S.A. Kahn, T.D. Azad, G. Sharareh, C.Y. Xu, L. Jie, A.S. Achrol, R. Chase, S. Pia. AntiCD47 Treatment Stimulates Phagocytosis of Glioblastoma by M1 and M2 Polarized Macrophages and Promotes M1 Polarized Macrophages In Vivo. Plos One. 2016;11:e0153550.

[11] T.M. Holling, E. Schooten, P.J.V.D. Elsen. Function and regulation of MHC class II molecules in Tlymphocytes: of mice and men. Hum Immunol.2004; 65 :1-290.

[12] M. Berlin, A. Fogdell-Hahn, O. Olerup, A. Eklund, J. Grunewald, HLA-DR predicts the prognosis in Scandinavian patients with pulmonary sarcoidosis. Am J Resp Crit Care . 1997;156:1601-1605.

[13] T. Løvig, S.N. Andersen, L. Thorstensen, C.B. Diep, G.I. Meling, R.A. Lothe, T.O. Rognum. Strong HLA-DR expression in microsatellite stable carcinomas of the large bowel is associated with good prognosis. Brit $\mathrm{J}$ Cancer. 2002;87:756-762.

[14] K. Sumiyoshi, H. Kuwano, M. Watanabe, M. Kitamura, Y. Toh, K. Sugimachi. HLA-DR antigen expression in squamous epithelial dysplasia and squamous cell carcinoma of the esophagus: an immunohistochemical study. Oncol Rep. 1999;6:301. 
[15] J. Li, Y. Xie, X. Wang, C. Jiang, X. Yuan, A. Zhang, C. Liu, L. Pang, F. Li, J. Hu. Overexpression of VEGF-C and MMP-9 predicts poor prognosis in Kazakh patients with esophageal squamous cell carcinoma. PeerJ. 2019;7 : e8182.

[16] T.A. Wynn, A. Chawla, J.W. Pollard. Macrophage biology in development, homeostasis and disease. Nature. 2013;496: 445-455.

[17] A. Mantovani, M. Locati. Orchestration of macrophage polarization. Blood. 2009;114:3135-3136.

[18] C. Jenny, H. Thorsten. Tumour-associated macrophages and cancer, Curr Opin Pharmacol. 2013;13 :595-601.

[19] M.J. Campbell, N.Y. Tonlaar, E.R. Garwood, H. Dezheng, H. Dan, Moore, A.I. Khramtsov, A. Afred, B. Frederick, C. Yinghua, D.O. Malaka. Proliferating macrophages associated with high grade, hormone receptor negative breast cancer and poor clinical outcome, Breast Cancer Res Tr.2011;128:703-711.

[20] N. Tonlaar, M. Campbell, E. Garwood, A. Khramtsov, D. Moore, A. Au, F. Baehner, D. Huo, M. David, O. Oluwasola. Association of Proliferating Macrophages with High Grade, Hormone Receptor Negative Breast Cancer. Cancer Res. 2009;69: 3042-3042.

[21] Winograd, Rafael, Evans, A. Rebecca, Bayne, J. Lauren, Vonderheide, H. Robert. CD40 immunotherapy for pancreatic cancer. Cancer Immunol Immun. 2013;62 :949-954.

[22] F. Qing-Min, J. Ying-Ying, Y. Guo-Feng, K. Xing-Rui, Y. Fei, G. Lu, L. Rong, Z. Qiu-Dong, Y. Yang, L. ZhengHua. Tumor-associated macrophages promote cancer stem cell-like properties via transforming growth factor-beta1-induced epithelial-mesenchymal transition in hepatocellular carcinoma. Cancer Lett. 2014;352 : 160-168.

[23] B.Z. Qian, J.W. Pollard. Macrophage Diversity Enhances Tumor Progression and Metastasis. Cell. $2010 ; 141: 1-51$.

[24] T. Zhao, W.H. Xia, M.Q. Zheng, C.Q. Lu, X. Han, Y.J. Sun. Surgical excision promotes tumor growth and metastasis by promoting expression of MMP-9 and VEGF in a breast cancer model. Exp Oncol. 2008;30:6064.

[25] Z.R. Li, Y.P. Li, M.L. Lin, W.R. Su, W.X. Zhang, Y. Zhang, L. Yao, D. Liang. Activated Macrophages Induce Neovascularization Through Upregulation of MMP-9 and VEGF in Rat Corneas. Cornea. 2012;31:10281035.

[26] F.O. Martinez, A.A. Sica, M. Locati. Macrophage activation and polarization. Front Biosci. 2008;13: 453461.

[27] C.M. Ohri, S. Aarti, R.H. Green, D.A. Waller, B. Peter. The tissue microlocalisation and cellular expression of CD163, VEGF, HLA-DR, iNOS, and MRP 8/14 is correlated to clinical outcome in NSCLC. Plos One. 2011;6 : e21874. 
[28] M.B. Palmer, A.A. Vichot, L.G. Cantley, G.W. Moeckel. Quantification and localization of M2 macrophages in human kidneys with acute tubular injury. Int J Nephrol. 2014;7 : 415-419.

[29] Yang JB, Zhao ZB, Liu QZ, Hu TD, Long J, Yan K, L. ZX. FoxO1 is a regulator of MHC-II expression and anti-tumor effect of tumor-associated macrophages. Oncogene. 2018;37: 1192-1204.

[30] Z. Wen, H. Liu, R. Gao, M. Zhou, J. Ma, Y. Zhang, J. Zhao, Y. Chen, T. Zhang, F. Huang, N. Pan, J. Zhang, B. Fox, H. Hu, L. Wang. Tumor cell-released autophagosomes (TRAPs) promote immunosuppression through induction of M2-like macrophages with increased expression of PD-L1. J Immuno Cancer. 2018;6:151.

[31] T.P. Miller, S.M. Lippman, C.M. Spier, D.J. Slymen, T.M. Grogan. HLA-DR (la) immune phenotype predicts outcome for patients with diffuse large cell lymphoma, J Clin Invest. 1988;82: 370.

[32] L.M. Rimsza, F. Pedro, D.A. Fuchs, M. Hamid, J.M. Connors, R.D. Gascoyne. HLA-DR protein status predicts survival in patients with diffuse large B-cell lymphoma treated on the MACOP-B chemotherapy regimen. Leukemia Lymphoma.2007; 48: 542-546.

[33] Liu J, Li C, Zhang L, Liu K, Jiang X, Wang X, Yang L, Liang W, Liu K, Hu J, L. F. Association of tumourassociated macrophages with cancer cell EMT, invasion, and metastasis of Kazakh oesophageal squamous cell cancer. Diagn pathol.2019; 14: 55.

[34] D.R. Rhodes, S. Kalyana-Sundaram, V. Mahavisno, R. Varambally, J. Yu, B.B. Briggs, T.R. Barrette, M.J. Anstet, C. Kincead-Beal, P. Kulkarni. Oncomine 3.0: Genes, Pathways, and Networks in a Collection of 18,000 Cancer Gene Expression Profiles. Neoplasia. 2007;9 : 166-180.

[35] D. Wankun, W. Yongbo, L. Zexian, C. Han, X. Yu. Heml: a toolkit for illustrating heatmaps. Plos One. 2014;9 : e111988.

[36] D.S. Chandrashekar, B. Bashel, S.A.H. Balasubramanya, C.J. Creighton, I. Ponce-Rodriguez, B. Chakravarthi, S. Varambally. UALCAN: A Portal for Facilitating Tumor Subgroup Gene Expression and Survival Analyses. Neoplasia.2017; 19: 649-658.

\section{Figures}



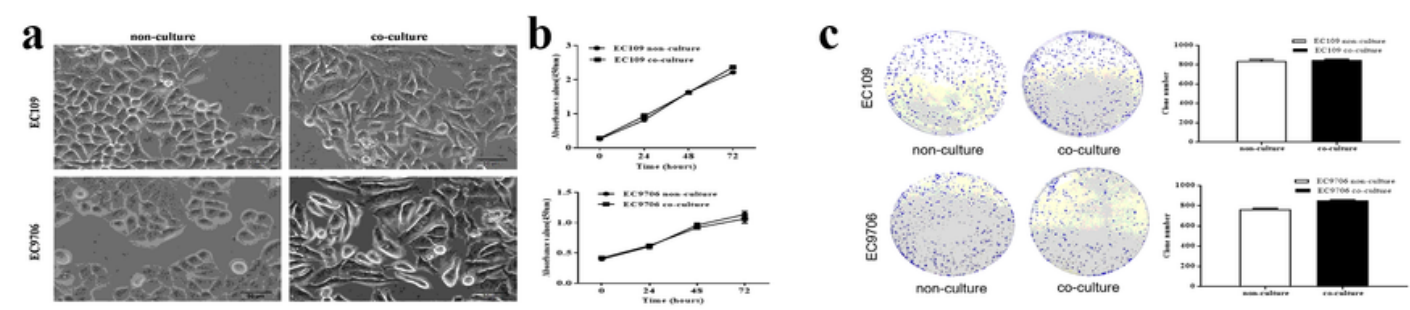

d
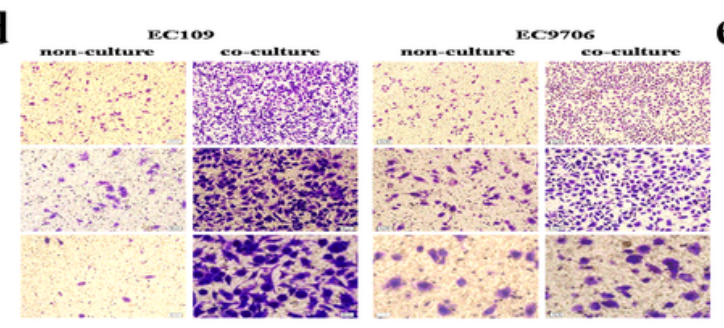

e
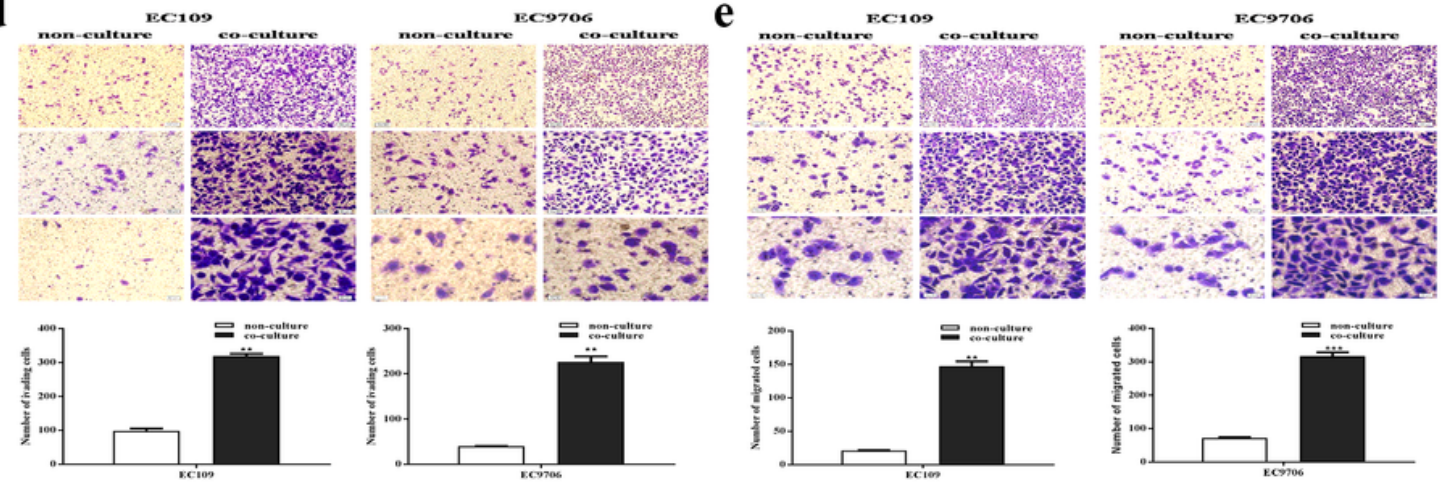

f

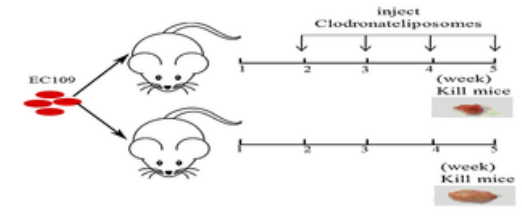

g
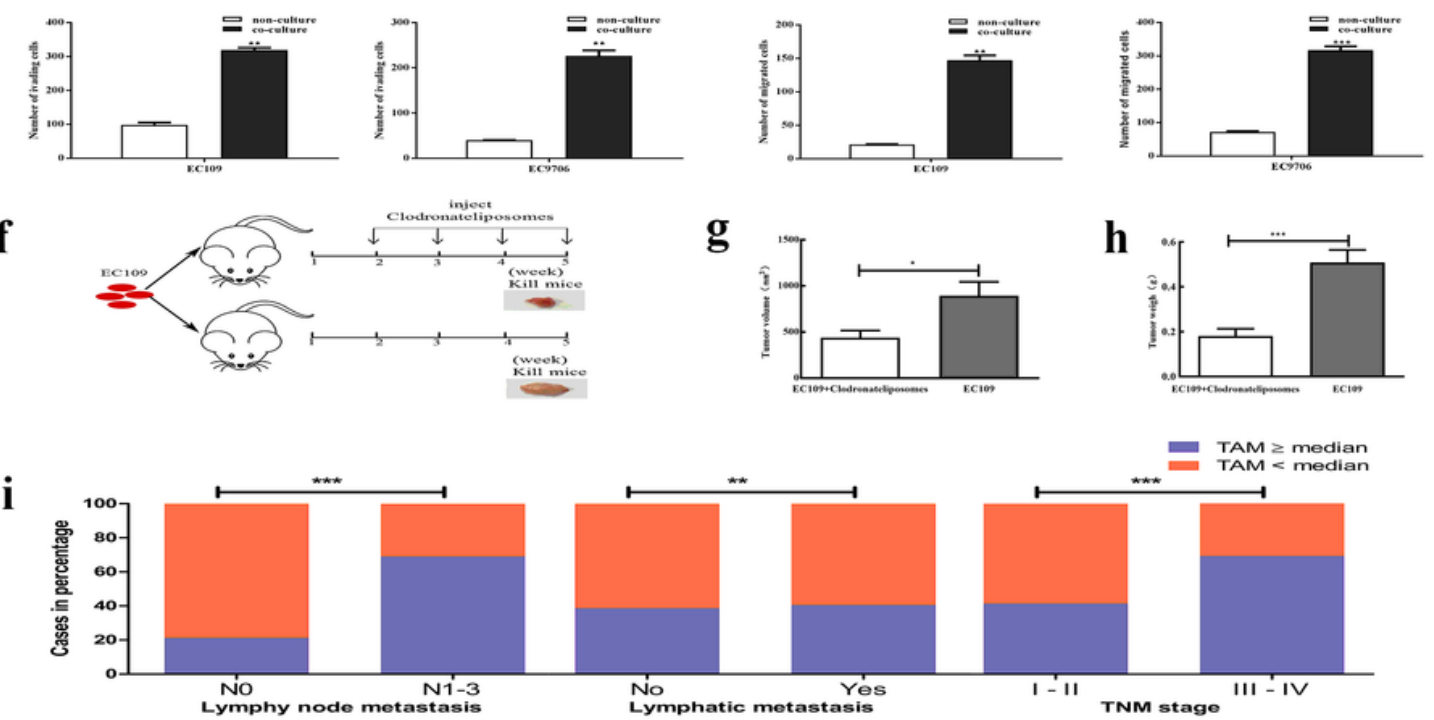

Figure 1

The effects of macrophages on the growth, invasion, and migration of esophageal squamous cell carcinoma (ESCC) cells. (a) The pattern of form EC109 and EC9706 were co-cultured with PMA-treated THP1 macrophages for $48 \mathrm{~h}$ (magnification, $\times 200$ ). (b-c) The effect of TAMs on the proliferation of EC109 and EC9706 cells via (b)CCK8 assay, (c) plate cloning experiment, TAM have no significantly effect on ESCC cells proliferation. (d-e) After EC109 and EC9706 co-culture with TAM or EC109 and EC9706 alone, (d) invasion experiment and (e) migration experiment were used to detect the migration and invasion capacity of EC109 and EC9706. (f) Schematic diagram of mice implanted with EC109 cells and injected with chlorate phosphate (depletion of TAMs). Differences in tumor volume (g) and weight (h) in BALB/c mice. (i) Relationship between CD68-positive TAMs and clinicopathological parameters in ESCC. TAM: tumor associated macrophage. 
a

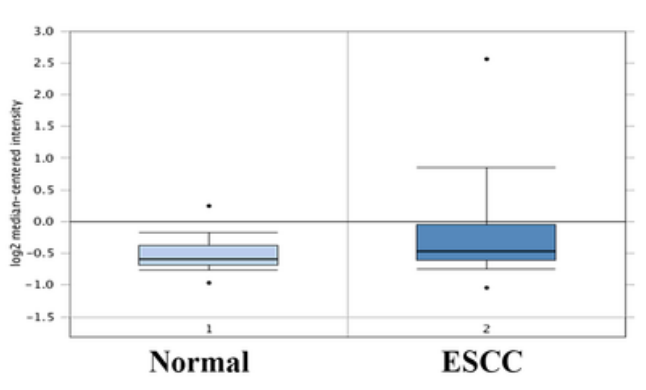

C
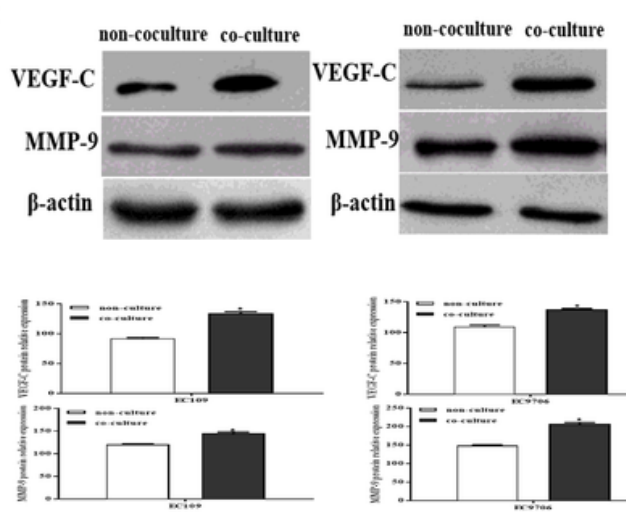

b

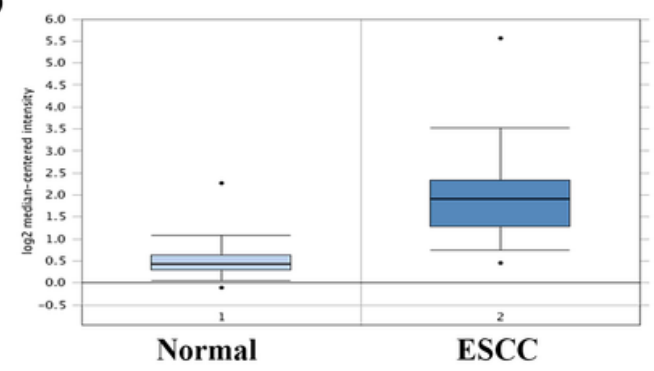

d
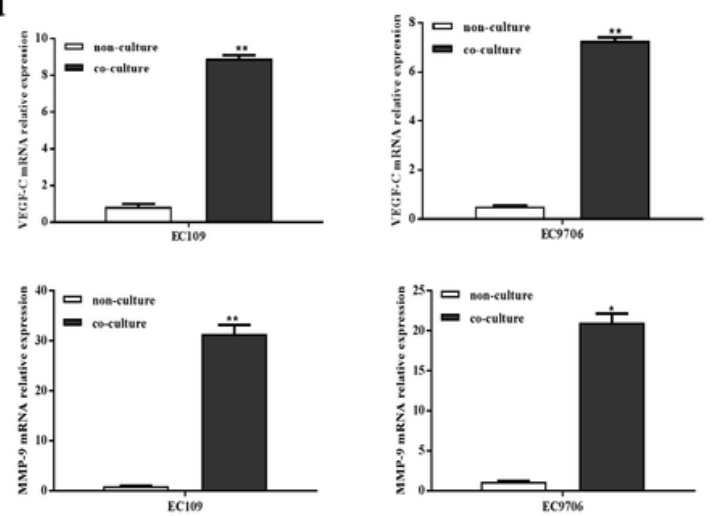

\section{Figure 2}

TAMs were an important source of VEGF-C and MMP-9 that promoted metastasis in ESCC tissue. (a-b) The Oncomine database was used to analyze the Su Esophagus dataset Compared with normal tissue, ESCC tissue exhibited (a) significantly higher expression of VEGF-C (P = 0.002; fold change $=1.264)$, and (b) significantly higher expression of MMP-9 ( $P=1.02 \mathrm{E}-13$; fold change $=2.787$ ). Macrophages co-cultured with EC109 cells exhibited significantly higher expression of the metastasis-related factors VEGF-C and MMP-9 at both the protein expression level (c) and mRNA expression level (d). 

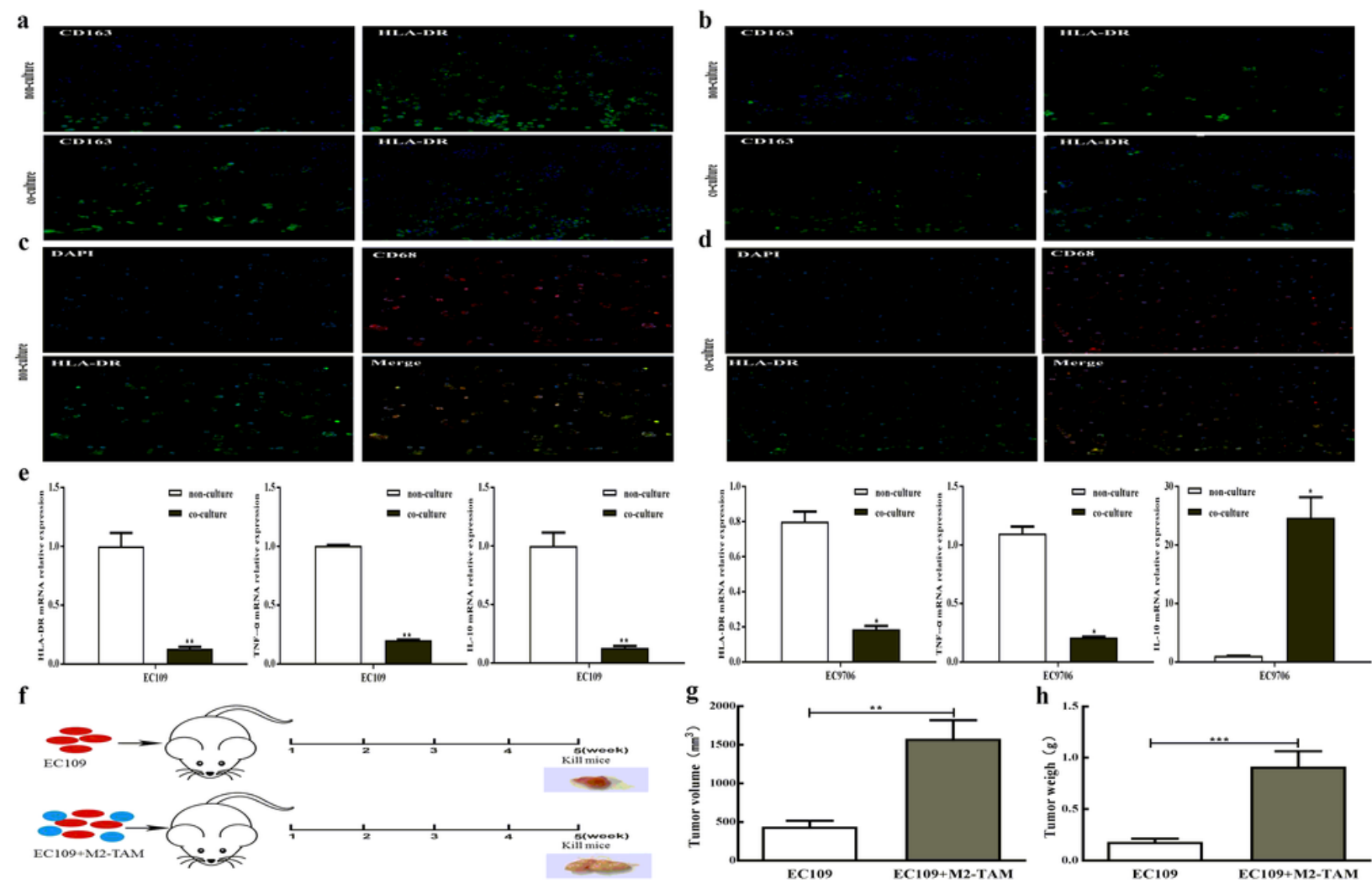

i

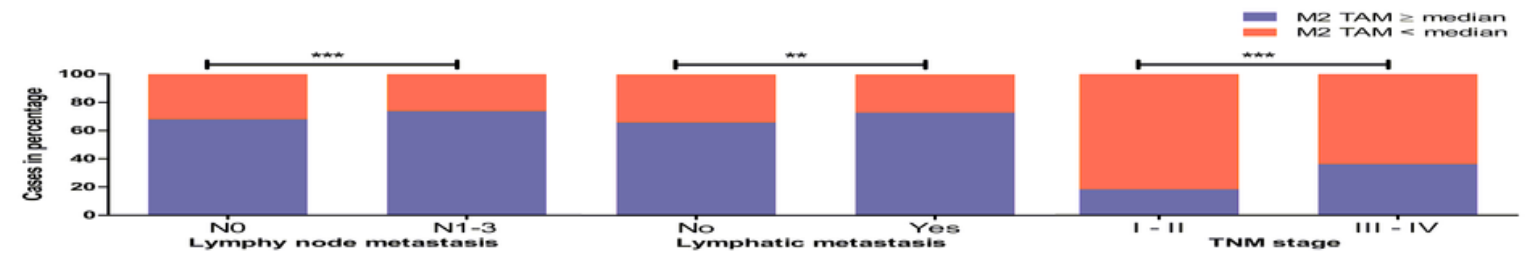

\section{Figure 3}

TAMs in ESCC were mainly M2 type, which promote metastasis and tumorigenicity of ESCC. (a) Immunofluorescence (IF) staining showed the expression of CD163 (M2 macrophages) and HLA-DR (M1 macrophages) in single-culture and co-culture with EC109/EC9706 cells and macrophages. Compared with single-culture, the number of CD163 macrophage-positive cells increased significantly in the co-culture setting, while the number of HLA-DR-expressing macrophages decreased significantly. (b) IF staining was used to detect the number of CD68 and HLA-DR double-expressing (M1 macrophage) cells before and after co-culture with EC109/EC9706 cells. Compared with single-culture, the number of CD68 and HLA-DR coexpressing cells was significantly reduced after co-culture. (c) qRT-PCR detection of gene expression indicative of macrophage phenotype changes after co-culture revealed a decrease in expression of the M1 type markers, HLA-DR and TNF-a, and an increase in expression of the M2 type marker IL-10. (d) Schematic diagram of mice co-implanted with EC109 cells and M2 macrophages, or with EC109 alone. Differences in tumor volume (e) and weight (f) in BALB/c mice. (g) Relationship between CD163-positive M2-TAMs and clinicopathological parameters of ESCC. 
a

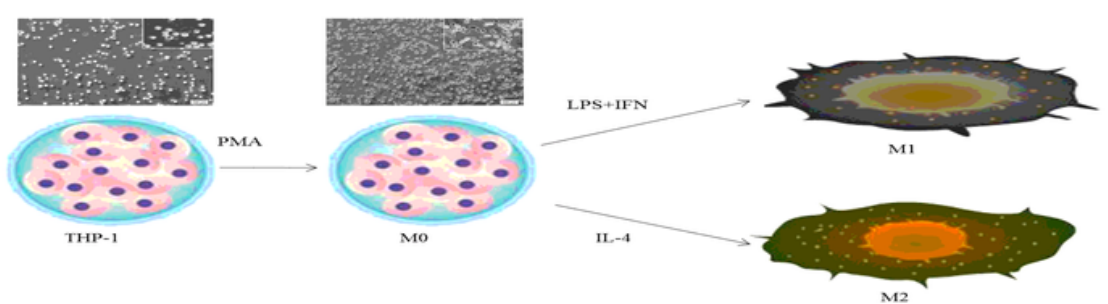

b

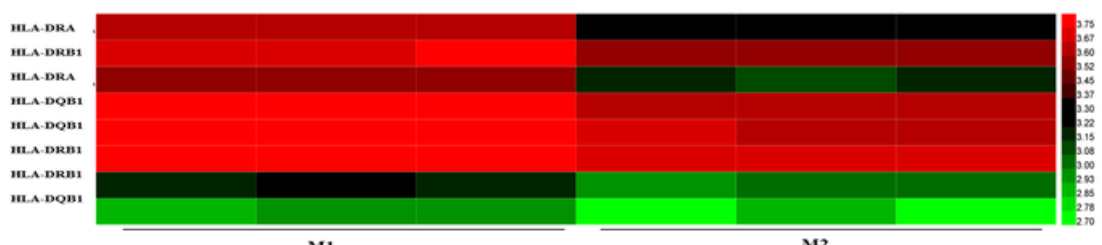

c
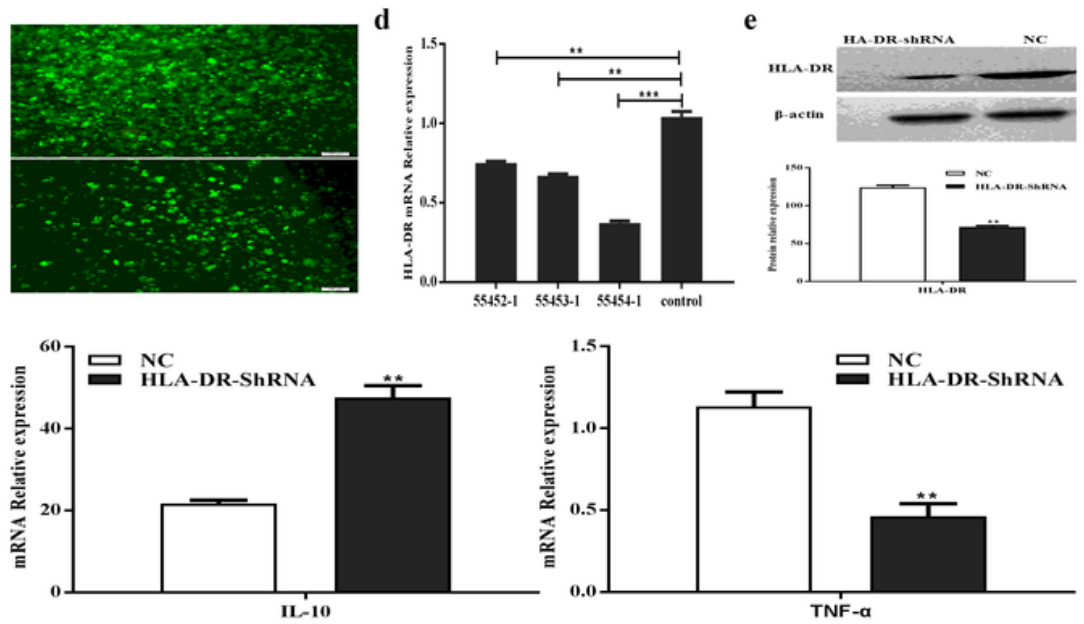

\section{Figure 4}

Knockdown of HLA-DR promoted macrophage transformation from the M1 to the M2 phenotype. (a) Schematic diagram of induction of THP-1 cells into M1 and M2 type macrophages, respectively. (b) Relative to M1 macrophages, expression of various members of the HLA-DR family was significantly downregulated in M2 macrophages (GEO: GSE95405), all data were standardized, and Hemap was used to visualize the heatmap. (c) Transfection efficiency of HLA-DR lentiviral shRNA-transfected macrophages. HLA-DRA-RNAi (55454-1)-transfected cells (green fluorescence) displayed by immunofluorescence after transfection of macrophages. qPCR (d) and Western blot (e) detection of three lentiviral shRNA and empty vector-treated control group indicated that macrophages transfected with HLA-DR shRNA exhibited reduced HLA-DR expression. (f) lentiviral shRNA transfection downregulated HLA-DR and influenced macrophage phenotype. 
a

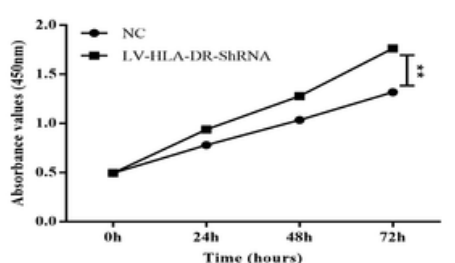

b

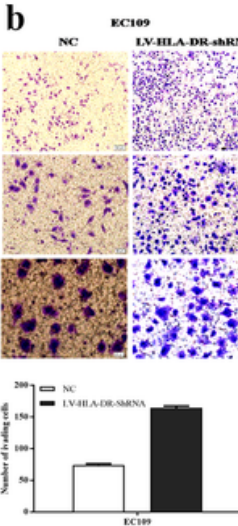

d

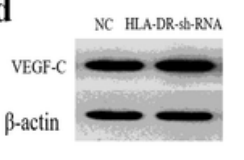

NC HLA-DR-sh-RNA
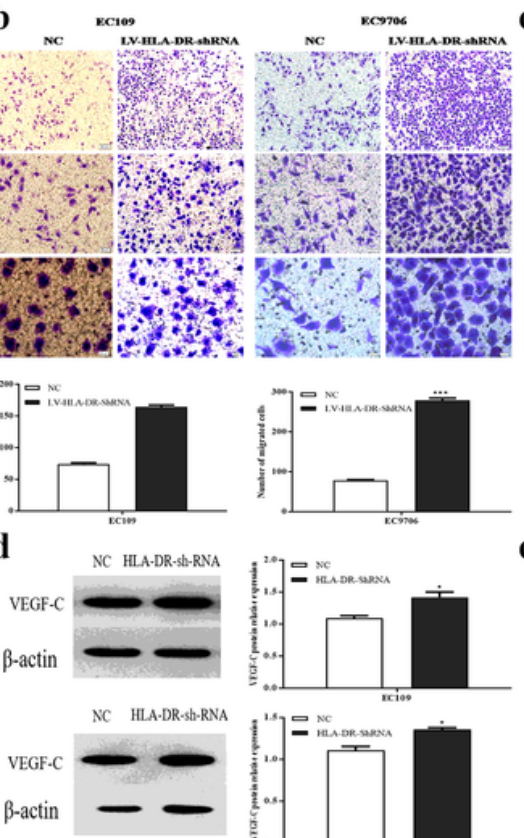
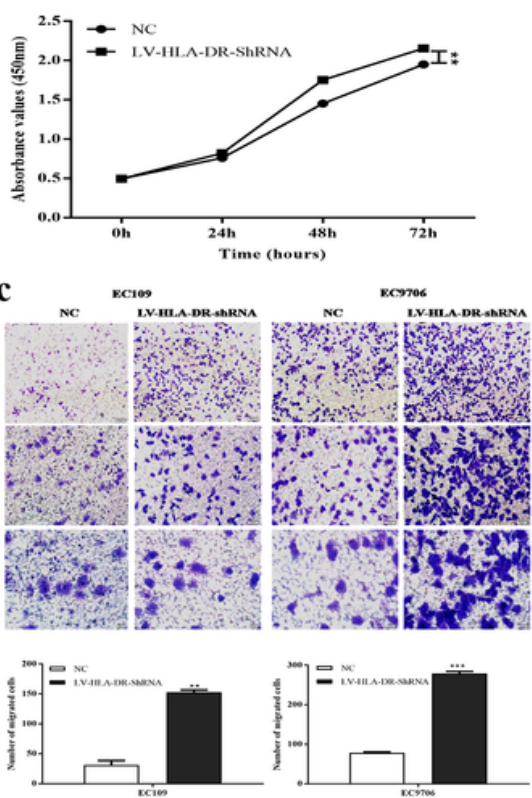

e

NC HLA-DR-Sh-RNA MMP-9 $\beta$-actin

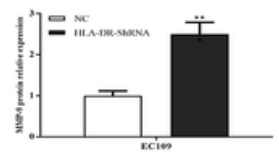

NC HLA-DR-sh-RNA

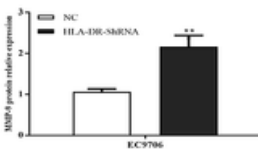

\section{Figure 5}

Knockdown of macrophage HLA-DR promoted proliferation, invasion and migration of ESCC. (a) Cell proliferation was higher in ESCC cells co-cultured with HLA-DR knockdown TAMs than in ESCC cells cocultured with macrophages transduced with the empty vector negative control (EC109 cells, left panel; EC9706 cells, right panel). Co-culture with HLA-DR knockdown TAMs promoted migration (b) and invasion (c) of ESCC cells. Knockdown of HLA-DR in TAMs induced (d) VEGF-C overexpression and (e) MMP-9 overexpression. 

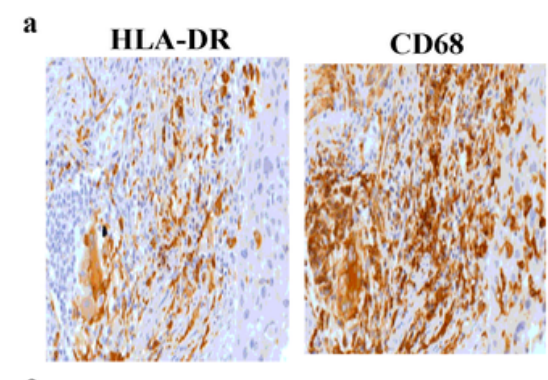

c

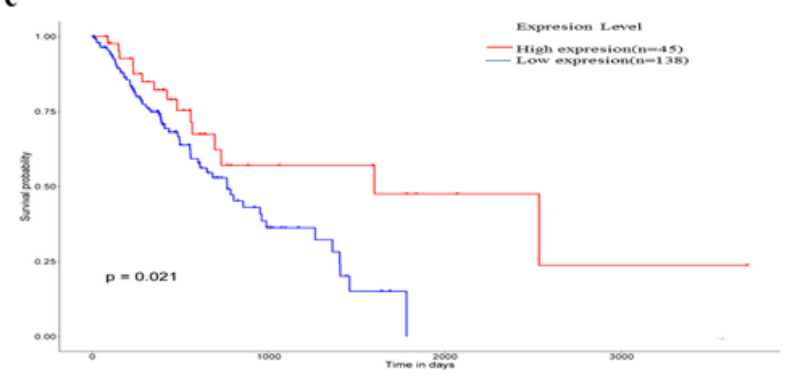

b

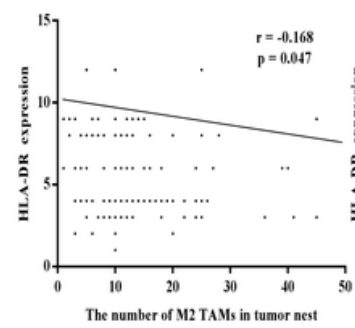

d

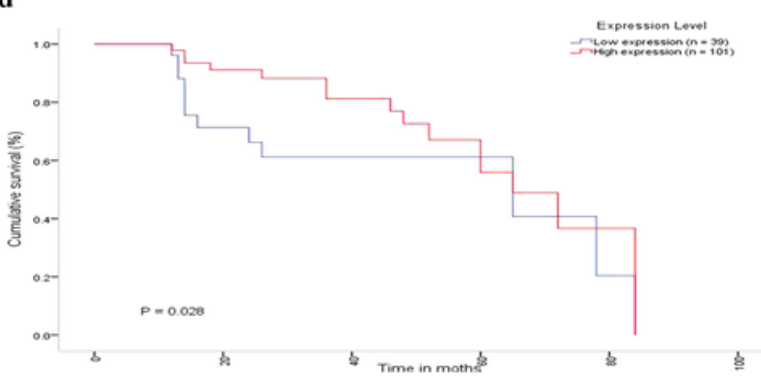

\section{Figure 6}

Low expression of HLA-DR is associated with low density M2 TAMs and poor prognosis in ESCC (a) HLADR is mainly expressed in stromal cells of ESCC, and macrophages are an important cellular component that express HLA-DR. (b) Lower expression of HLA-DR was positively correlated with an increase in the number of M2 TAMs. (c) Analysis of data from the TCGA database demonstrated that lower expression of HLA-DR was associated with poor outcomes in patients with ESCC. (d) Low HLA-DR expression is closely related to poor prognosis in patients with Kazakh ESCC.

\section{Supplementary Files}

This is a list of supplementary files associated with this preprint. Click to download.

- SupplementaryTable1.docx

- SupplementaryTable1.docx 